\title{
Acquisition Dependent Random Access for Connectionless CDMA Systems
}

\author{
Aylin Yener \\ Roy D. Yates \\ Wireless Information Network Laboratory (WINLAB), Rutgers University \\ yener@winlab.rutgers.edu ryates@winlab.rutgers.edu
}

\begin{abstract}
We consider connectionless packet switched CDMA systems where all users have to share a common bandwidth and signature sequence on a contention basis. Multiple access is achieved by the simultaneous acquisition of different users' spread spectrum transmissions. The throughput of a pure random access system of this kind suffers from acquisition errors and timing mismatches of the active users as well as instability issues. In this paper, we consider schemes that use information available at the physical layer, i.e., at the output of the multiuser access detector (MUAD) designed for this random access system, to improve and stabilize the throughput. We consider timing randomization, pseudo-Bayesian stabilization and collision resolution algorithms and report the corresponding numerical results.
\end{abstract}

\section{Introduction}

Code Division Multiple Access (CDMA) has a number of advantages such as flexibility in frequency planning and its resistance to jamming and frequency selective fading and has emerged as a promising candidate for third generation systems $[1,2]$. A variety of services are envisioned for third generation and future wireless systems including packet switching based architectures. The services inevitably include applications where a relatively large user population needs to be served, but each user needs to send short data packets occasionally. Such an application is the basis for using a random access mechanism for efficient use of system resources where the resources are to be shared among all the users on a contention basis as opposed to being uniquely assigned to each user. In this work, we consider packet switched CDMA systems for short message services where random access is employed in a wide band system employing CDMA.

A packet switched CDMA system has a connectionless architecture, i.e. no connection is to be kept between a user and the base station except when the user is sending information. Thus, user recognition and synchronization must be established every time a user needs to transmit information. It is a random access system where users have to contend for resources. Thus, users do not have assigned signature sequences and have to try sending information using one of a set of predetermined signature sequences.

Considerable effort has been directed towards modeling and analyzing random access CDMA systems. While CDMA slotted ALOHA systems assume a unique signature for each user randomly accessing the system [3-6], spread ALOHA assumes a single signature shared by all users [7-9]. Existing analyses (e.g. [3-9]) often ignore the fact that user acquisition (user recognition and timing acquisition) has to be achieved for every transmission or state that it can be achieved readily and thus assume the system to be interference limited after access has been achieved by all active users. On the other hand, for circuit switched CDMA, there is a body of research that concentrates on user parameter acquisition (e.g. [10]) and it has been well known that timing acquisition can be capacity limiting [11].

Recently, the capacity of short message packet switched CDMA systems has been investigated taking into account the fact that user acquisition has to be achieved during each information transfer session, e.g. each packet or frame, [12,13]. The model adopted is that of a random access CDMA system where a single access signature is available as in spread ALOHA. Note that although all users use the same signature it is possible to serve multiple users. Specifically, we employ a slotted system. However, within a slot, the exact instance of arrival of a packet transmission for each user is different. We refer to the difference between the arrival time and the beginning of the slot time as the timing delay of the user. Multiple users can be resolved if the timing delays of the users are sufficiently apart. To reduce ambiguity, we use the term packet delay for the time it takes for the packet to be transmitted successfully from the time instance it is generated. Packet delay is the random access delay of the system and is a result of contention and queueing. To keep packet delay small, it is desirable to use short data packets containing perhaps a few hundred data bits. Furthermore, efficiency dictates that the acquisition overhead be small in comparison to the number of information bits. Consequently, the multiaccess capacity of such a communication system is access efficiency limited rather than interference limited [13].

In this paper, we investigate capacity enhancement techniques at the MAC layer for the same connectionless CDMA system taking the physical layer conditions of this system into account. First, we consider a simple transmission strategy where each user effectively adjusts its packet arrival time to be uniform in a larger delay space than what is defined by the maximum propagation delay in the system to increase the 
maximum achievable throughput. We then consider collision resolution methods to stabilize the performance of this random access system. The common feature of each of these algorithms is the fact that each of them would be using the information available about the number of users at the output of the multiuser access detector proposed in [12] to implement the corresponding algorithm. We describe the algorithms and evaluate the performance of each for a sample system.

\section{Communication Model}

We consider a slotted CDMA system where multiple users can attempt to access the system at the same time. The communication format is that each user sends a packet which consists of a preamble sequence (a sequence of $1 \mathrm{~s}$ ) followed by the information content of the packet. Each bit in the packet (both preamble and information bits) are modulated by the same access signature sequence for each user. We assume that all potential users have acquired the base station's pilot signal and are tuned to a downlink paging channel where they can receive broadcast messages. Furthermore, we assume a TDD system and that each user has acquired its downlink and thus uplink gain. This enables each user to adjust its transmit power so that all users are received with equal power at the base station. The start time of each slot is broadcasted from the base station along with other necessary access parameters. The timing delay uncertainty of the new users thus comes from their round trip propagation delays relative to the broadcast of the base station. We assume these delays to be less than 1 bit period for each new user (see Figure 1).

The base station has to detect the activity of a random number of users along with the timing delays of each of these users at each slot to be able to decode the packets of the active users. In [12], this is called the "multiuser access detection" problem. While the major difference between multiuser access detection and the multiuser timing acquisition is the uncertainty about the activity status' of all the users, one should also note the stringent requirement on the time frame in which the access has to be completed, i.e., the acquisition time should be much less than the duration the information bits which is likely be on the order of a few of hundreds of bits. Once the user's presence is detected by the system, the base station attempts to decode the packet of this user. If the packet is decoded successfully, the user receives an acknowledgment. If the user fails to receive an acknowledgment, the packet is lost and either discarded or queued at the user's transmitter to be retransmitted.

A two-stage receiver whose initial stage works on the transmitted preambles to detect the activity status of the users and is followed by a detector which will decode the active users' information bits using the findings of the first stage is proposed in [12] (Figure 2). The performance of the first stage, called the Multiuser Access Detector (MUAD), is of vital importance to the system since the performance of the second stage detector hinges upon the correctness of the information supplied by the first stage. A false alarm event, the event that the system erroneously declares a user present when there is none, implies a waste of resources for the second stage since it may require the detector to try to decode fictitious users and to try to suppress the corresponding nonexistent interference to other users. A miss event, the event that the system fails to capture a user, is also highly undesirable since an active user will not enter the system and its interference to the other users will not be cancelled during the second stage.

Given the assumptions above, the received signal during the first stage of access is

$$
r(t)=\sum_{i=1}^{N_{A}} \sqrt{q_{i}} s_{a}\left(t-\tau_{i}\right)+n(t) \quad t \in\left[0, L T_{b}\right]
$$

where $N_{A}$ is the number of active users, $q_{i}$ and $\tau_{i}$ are the received power and the timing delay of the user $i$, and $n(t)$ is the zero mean white Gaussian noise with power spectral density $\sigma^{2}, L>1$ is the length of the preamble in bits, and $T_{b}$ is the bit duration. We assume $0 \leq \tau_{i} \leq T_{\max }<T_{b}$ and express $\tau_{i}$ as $\tau_{i}=\left(k_{i}+\alpha_{i}\right) T_{c}$ where $k_{i}=\left\lfloor\tau_{i} / T_{c}\right\rfloor$. When $k_{i}=k$, we say that user $i$ is in interval $k$.

The accessing signature sequence $s_{a}(t)$ is

$$
s_{a}(t)=\sum_{i=0}^{G-1} c(i) \frac{1}{\sqrt{G}} p\left(t-i T_{c}\right)
$$

where $G$ is the processing gain, $T_{c}$ is the chip duration, $c(i) \in$ $\{-1,1\}$ is the $i$ th chip value, and $p(t)$ is the rectangular chip waveform normalized to have unit energy. If the received signal is chip matched filtered and sampled at the chip rate from the start of the slot with 1-bit delay, thus observed for a total of $L-1$ bits, the discretized received signal in the $j$ th observation interval $\left(\left[j T_{b},(j+1) T_{b}\right]\right)$ can be expressed as [13]

$$
\mathbf{r}_{j}=\mathbf{S v}+\mathbf{n}_{j}
$$

where $\mathbf{n}_{j}$ is a white Gaussian vector, $\mathbf{S}$ is the matrix with columns $\mathbf{s}_{0}, \ldots, \mathbf{s}_{M-1}$ and $\mathbf{s}_{j}$ is the signature sequence circularly shifted by $j$ chips, i.e. $\mathbf{s}_{0}=[c(0), \cdots, c(G-1)]$ and $\mathbf{s}_{j}=[c(G-j), \cdots, c(G-1), c(0), c(1), \cdots,(G-1-j)]$ for $1 \leq j<G$. The vector $\mathbf{v}$ is

$$
v_{i}= \begin{cases}\sum_{k \in B_{i}}\left(1-\alpha_{k}\right) \sqrt{q_{k}}, & i=0 \\ \sum_{k \in B_{i-1}} \alpha_{k} \sqrt{q_{k}}+\sum_{k \in B_{i}}\left(1-\alpha_{k}\right) \sqrt{q_{k}}, & 1 \leq i<M-1 \\ \sum_{k \in B_{i-1}} \alpha_{k} \sqrt{q_{k}}, & i=M-1\end{cases}
$$

where $B_{i}$ as the set of users in interval $i$. The decorrelating multiuser access detector (DMUAD) [13] works on $\mathbf{r}=\frac{1}{L-1} \sum_{j=1}^{L} \mathbf{r}_{j}$, and eliminates users that are outside $[(i-$ 
2) $\left.T_{c}, i T_{c}\right]$ at each detector output $y_{i}$. The output of the detector is

$$
\mathbf{y}=\frac{1}{(L-1)}\left(\mathbf{S}^{\top} \mathbf{S}\right)^{-1} \mathbf{S}^{\top} \mathbf{r}=\mathbf{v}+\tilde{\mathbf{n}}
$$

where $\tilde{\mathbf{n}}$ is $\mathbf{0}$-mean Gaussian noise vector with covariance $\frac{\sigma^{2}}{(L-1)} \Gamma^{-1}$ where we denote $\Gamma=\mathbf{S}^{\top} \mathbf{S}$. In general, $v_{i}$ contains contributions from an unknown number of users each of which has an unknown delay offset, and it is difficult to solve for the exact number of active users. Instead, DMUAD tries to detect if there are any active users around the $i$ th delay value by designing a simple threshold test [12]. If activity is detected at the output of the $i$ th decorrelator, the second stage detector should use this information to try and decode the identification information of a user around the corresponding timing delay value. The second stage detector, as explained before is a multiuser bit detector.

The above filtering operation has a by-product that will prove to be useful in the sequel. It is easy to see that the sum of the DMUAD outputs can be expressed as

$$
z=\sum_{i=1}^{M} y_{i}=N_{A} \sqrt{q}+\eta
$$

where $\eta=\sum_{i=1}^{M} \hat{n}_{i}$ is the sum of $M$ correlated Gaussian random variables. Thus

$$
\hat{N}_{A}=\operatorname{round}(z / \sqrt{q})
$$

is the unbiased ML estimate for the number of users accessing the system. Thus, we can use this information at the MAC layer to design efficient access algorithms as explained next.

\section{Capacity Enhancement Issues}

In this section, we investigate several techniques to enhance the capacity of the connectionless packet CDMA communication system. We assume that the system has an infinite user population, a Poisson number of new arrivals per slot with mean $\lambda$ and that this mean value is constant (or slowly varying). The two-stage multiuser access receiver with DMUAD first stage is used at the base station and we assume that the base station obtains an estimate of the number of users attempting to access as given by (7). For each of the following methods, the common thread is that the mobile or the base station utilizes features specific to the physical layer design of this system to improve the system performance at the MAC layer.

The first improvement we consider is a simple transmit strategy that users can implement to improve the capacity of this packet switched CDMA system. Reference [13] showed that the capacity of the packet switched CDMA system is low due to the fact that packets belonging to different users that are arriving at the base station collide in propagation delay space. That is, when two or more users' signals arrive with similar timing delays, the individual delays and consequently the users cannot be resolved by the MUAD. The simplest way of minimizing this type of collisions is to disperse the users in timing delay space as much as possible, i.e. let the users transmit their signals so that the arrival time distribution is expanded to the whole bit interval. Since we assume a TDD system and that each user has already acquired the downlink, the users have a good estimate of their propagation delays, and thus send their signals with a randomized delay value between $\left[0, T_{b}\right]$ with the appropriate offset for their true propagation delay, such that each user has a delay at the base station that is uniform in $\left[0, T_{b}\right]$. This would require $\mathbf{S}$ to include all possible integer circular shifts of the access signature sequence, and a $G \times G$ matrix $\mathbf{S}^{\top} \mathbf{S}$ to be inverted. The resulting y has $G$ elements each of which needs to be compared against the DMUAD threshold [12]. Although some asymptotic efficiency loss is to be expected by the larger matrix inversion [14], the benefit of having more filter outputs should in general overpower this loss, especially when an access signature with good auto-correlation properties is used.

Now we examine the retransmission of backlogged packets (users) resulting from unsuccessful transmissions. In this case, similar to ALOHA, the packet switched CDMA system also suffers from instability. In particular, the number of transmitting users per slot has to be stabilized so that the system does not become overloaded due to the retransmissions as well as new attempts. In what follows, we suggest the use of two protocols which stabilize the packet switched CDMA system. The reason for studying these particular algorithms is the fact that they both are designed utilizing the information supplied by the receiver used at the physical layer, i.e., DMUAD.

First we consider the well known Pseudo-Bayesian stabilization algorithm for slotted ALOHA $[15,16]$. The algorithm, designed for the pure collision channel, assigns a permission probability to all packets, new or backlogged, equal to $1 / b$ where $b$ is the estimated number of backlogged packets. The idea is to try to keep 1 user active at a time to minimize the collisions. A straightforward extension of this algorithm can be designed for the packet switched CDMA system. Since multiple users can be resolved at a time, the permission probability should be adjusted such that the number of users active in the system maximizes the throughput of the system. In particular, the base station can broadcast the permission probability $m / b$, where $m$ is chosen such that the system throughput is maximized when $m$ users are present in the system. The value $m$.is highly dependent on the performance of the physical layer as demonstrated in the next section. The number of backlogged users can be estimated by the base station by keeping a count of the estimate of the number of active users $\left(\hat{N}_{A}\right)$ users minus the users that successfully transmit in a given slot.

Next let us consider the first come first serve (FCFS) splitting algorithm for the 0,1 , collision channel [15]. In this system, each packet is transmitted according to its timestamp. The base station tracks a time $t_{r}$ such that it is certain that all 
packets timestamped prior to $t_{r}$ have been successfully transmitted. Starting with time $t_{r}$, the base station announces an allocation interval such that all packets with timestamps in that interval are transmitted. The FCFS splitting algorithm is designed for a single capture (pure collision) channel, and assumes the receiver is able to detect a collision as well as an idle channel. If a collision is detected, a new allocation interval that is half the size of the previous is announced, splitting the set of packets involved in the collision in two sets. When a success or idle is detected, $t_{r}$ is advanced to the end of the allocation interval and a new allocation interval is announced.

The FCFS algorithm can be modified so that it accounts for the fact that the system can resolve more than one users in a slot and applied to stabilize the packet switched CDMA system as follows. A collision for the packet switched CDMA system is defined as the event that at least one of the users (packets) which attempt to access does not succeed in the given allocation interval. Thus, if the same allocation interval is enabled following a collision, the number of users transmitting in the interval is at most the same as the previous attempt. At this point one can use timing delay randomization, the first method explained in this section, to further disperse the remaining users in this interval for better detection and the remaining users may be able to get through without the need for splitting the allocation interval, thus avoiding unnecessary splitting and access delays for the users in this allocation interval. One simple modification to account for the capture effect is to let all packets involved in a collision to retry some number of times before the time interval is split as opposed to splitting the interval immediately following a collision as demonstrated by the numerical results in the next section. Once again, the information needed to implement of this algorithm, i.e. collision and idle detection in an allocation interval is readily available at the base station using $\hat{N}_{A}$.

\section{Results}

The capacity of the packet switched CDMA system is a function of the number of successful users given the number of active users which depends on the performance of the MUAD and the performance of the second stage bit detector which clearly is coupled with that of the MUAD under given system parameters which include the user arrival rate, MUAD false alarm rate and the quality of service requirement for reliable connection to the system. Since the exact analytical expression for the success probability of a user is complex, we have simulated a DS/CDMA system with processing gain $G=7$ to observe the performance of this system. The signature sequence is chosen to be an $m$-sequence of length 7 . We assume equal received power for all users at $20 \mathrm{~dB}$ and the background noise power $\sigma^{2}$ to be unity $(0 \mathrm{~dB})$. In all experiments, the thresholds for the decorrelator outputs of the first stage are set such that the false alarm rate of each test is $\alpha_{F}=0.01$. The second stage receivers constructed are one-shot asynchronous decorrelators or matched filters. For all experiments, the reliable connection requirement is that the signal-to-interference ratio (SIR) of the user has to be above $5 \mathrm{~dB}$. The propagation delays are assumed to be all less than 3 chips, however, the timing delays of all users are assumed to be uniform between $\left[0, T_{b}\right]$, i.e. timing delay randomization is employed by each user.

Figure 3 shows the average number of users the system can support, i.e. the throughput, versus the Poisson arrival rate in the absence of retries. The figure compares the throughput for different multiuser access receivers. In particular, we compare having a delay tracking algorithm versus no tracking algorithm following the DMUAD as well as having a matched filter versus an asynchronous decorrelator in the bit detection (second) stage of the multiuser access receiver. In [13], it was shown that, even in the presence of perfect tracking, where a user's timing delay is assumed to be tracked perfectly if this user is the sole arrival in a chip interval, essentially avoiding delay mismatches in the second stage of the multiuser access receiver, the capacity of this system is less than that of sum capacity of $G$ parallel ALOHA channels occupying the same bandwidth of the packet switched CDMA system (Figure 3). Figure 3 also shows the capacity of a system where all users arrive in a chip synchronous fashion, essentially avoiding the fractional chip delay mismatches in both stages of the multiuser access receiver. As expected, this scenario yields a tighter upper bound for the perfect tracking capacity than that of the ALOHA bound. It is notable that, each receiver, depending on its tracking capabilities and the kind of bit detector used in the second stage of the multiuser access receiver, yields the maximum throughput at a different arrival rate. For example, when a decorrelator and perfect tracking is used the maximum throughput is achieved at $\lambda=5$ whereas a matched filter with no tracking achieves its capacity at $\lambda=3$.

Figure 4 shows the improvement achieved by randomizing the propagation delays to the entire bit interval. The original maximum propagation delay of this system is $T_{\max }=3 \mathrm{chips}$ and the bit interval is 7 chips long. Both the decorrelator and the matched filter based receivers are able to resolve more users with timing delay randomization.

Next, we consider the stabilization of the system when unsuccessful packets are backlogged and retransmitted until they are successful. The pseudo-Bayesian stabilization algorithm is employed using different permission probabilities for different receivers. Figure 5 shows the average packet delay of a user when the decorrelator with no tracking and the decorrelator with perfect tracking are stabilized using a permission probability equal to $m / b$ where $b$ is the size of the backlog and $m=4$ for the no tracking case and $m=5$ for the perfect tracking case. Note that $m$ is chosen as the arrival rate that maximizes the throughput for both cases. As expected, the packet delay becomes unbounded beyond arrival rates that are the corresponding maximum throughput values in Figure 3. We also implemented variants of the FCFS splitting algorithm when 2 or 4 retries are allowed before splitting an interval and 


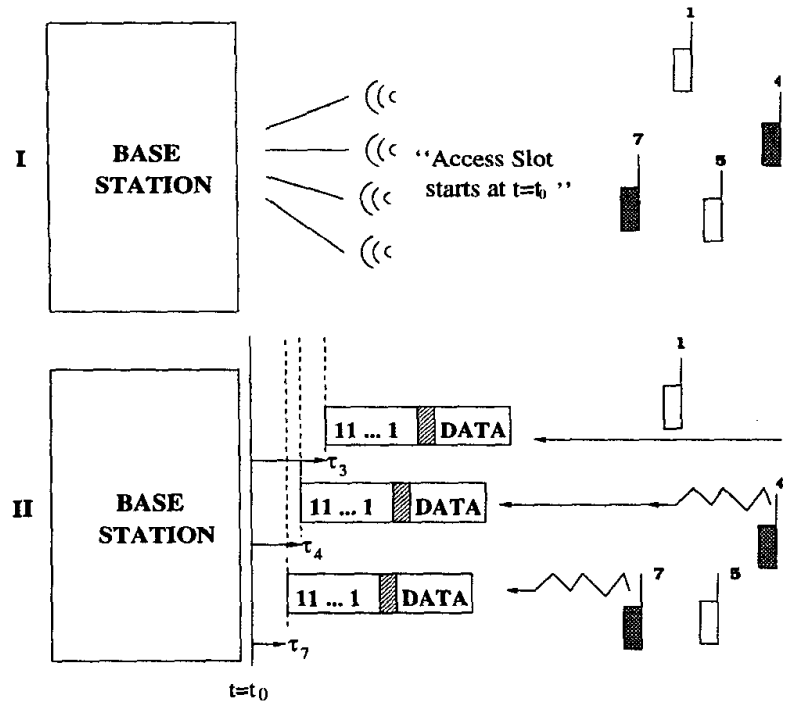

Figure 1: Multiaccess Model for packet switched CDMA

observed similar results (Figure 6).

In this paper, we investigated MAC layer solutions for enhancing the performance of a connectionless packet switched CDMA system. The physical layer of this system is observed to be limited by the multiuser acquisition efficiency and one needs to design the MAC layer keeping in mind the performance of the physical layer and the corresponding multiuser access receiver. We studied and presented results of throughput improvement/stabilization algorithms that use the information available from the physical layer regarding the current load of the system and that are exclusively depend on the performance of the physical layer.

\section{References}

[1] E. Dahlman, B. Gudmundson, M. Nilsson, and J. Skold. UMTS/IMT-2000 based on wideband CDMA. IEEE Communications Magazine, 36(9):70-80, September 1998.

[2] The cdma2000 ITU-R RTT candidate submission.

[3] D. Raychaudhuri. Performance analysis of random access packet-switched code division multiple access systems. IEEE Transactions on Communications, 29(6):895-901, June 1981.

[4] P. W. de Graaf and J. S. Lehnert. Performance comparison of a slotted ALOHA DS/SSMA network and a multichannel narrow-band slotted ALOHA network. IEEE Transactions on Communications, 46(4):544-552, April 1998.

[5] R. D. J. van Nee, R. N. van Wolfswinkel, and R. Prasad. Slotted ALOHA and code division multiple access techniques for land-mobile satellite personal communications. IEEE Journal on Selected Areas in Communications, 13(2):382-388, February 1995.

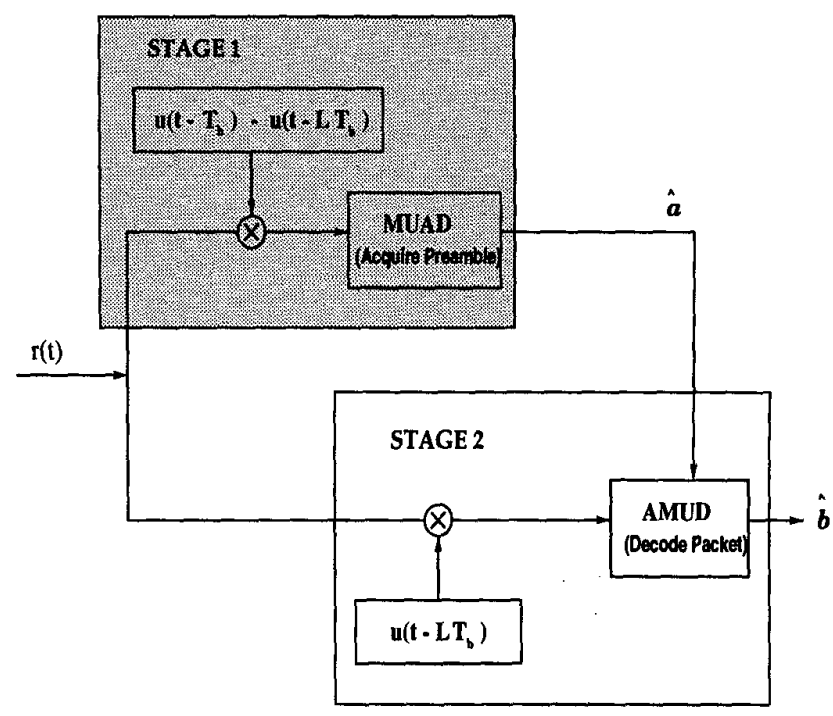

Figure 2: Two-stage receiver for packet switched CDMA

[6] Code division multiple access networks - IV. IEEE Journal on Selected Areas in Communications, 14(9), December 1996.

[7] N. Abramson. Fundamentals of packet multiple access for satellite networks. IEEE Journal on Selected Areas in Communications, 10(2), February 1992.

[8] F. Khan, C. Roobol, and J. Larsson. Performance of a common channel packet access in WCDMA. In IEEE PIMRC 98', 1998.

[9] N. Guo, S. D. Morgera, and P. Mermelstein. Common packet data channnel (CPDC) for integrated wireless DS-CDMA networks. IEEE Journal on Selected Areas in Communications, 14(4):735-749, May 1996.

[10] S. E. Bensley and B. Aazhang. Subspace-based channel estimation for code division multiple access communication systems. IEEE Transactions on Communications, 44(8):10091020, August 1996.

[11] U. Madhow and M. B. Pursley. Acquisition in direct-sequence spread-spectrum communication networks: An asymptotic analysis. IEEE Transactions on Information Theory, 39(3):903-912, May 1993.

[12] A. Yener and R. D. Yates. Multiuser access detection for CDMA systems. In Conference on Information Sciences and Systems (CISS'98), pages 17-22, March 1998.

[13] A. Yener and R. D. Yates. Multiuser access capacity of packet switched CDMA systems. In VTC'99, pages 1846-1850, May 1999.

[14] S. Verdú. Multiuser Detection. Cambridge University Press, 1998.

[15] D. Bertsekas and R. Gallager. Data Networks. Prentice-Hall, 1987.

[16] R. L. Rivest. Network control by Bayesian broadcast. Technical Report MIT/LCS/TM-285, MIT, Laboratory for Computer Science, Cambridge, MA, 1985. 


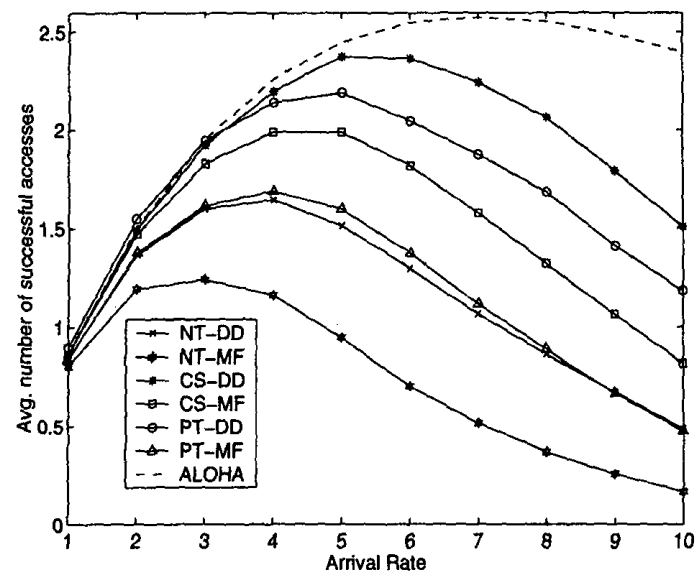

Figure 3: Comparison of access capacity for different scenarios: NT:No tracking, PT: Perfect Tracking, CS: Chip Synchronous, DD: One-shot decorrelator, MF: Matched Filter for second stage receivers, ALOHA: $M=7$ orthogonal pure RA channels. No retries.

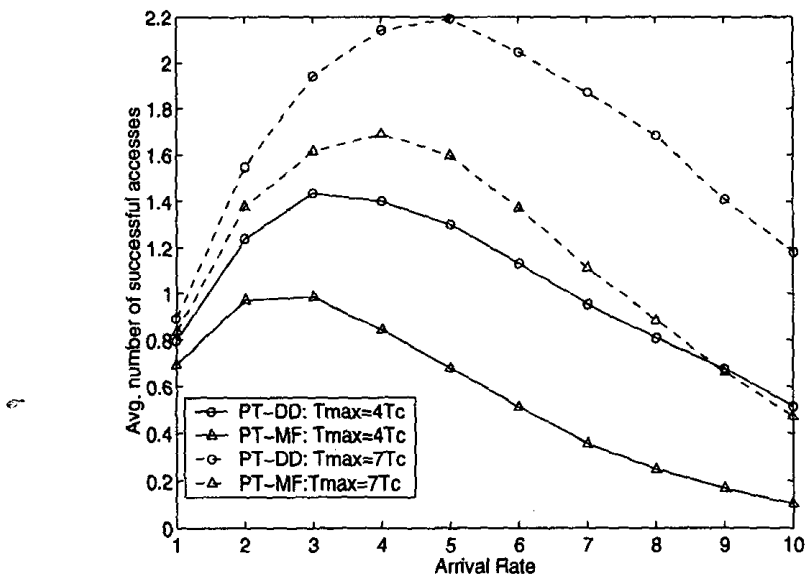

Figure 4: Comparison of access capacity for $T_{\max }=3 T_{c}$ and $T_{\max }=7 T_{c}$. No retries.

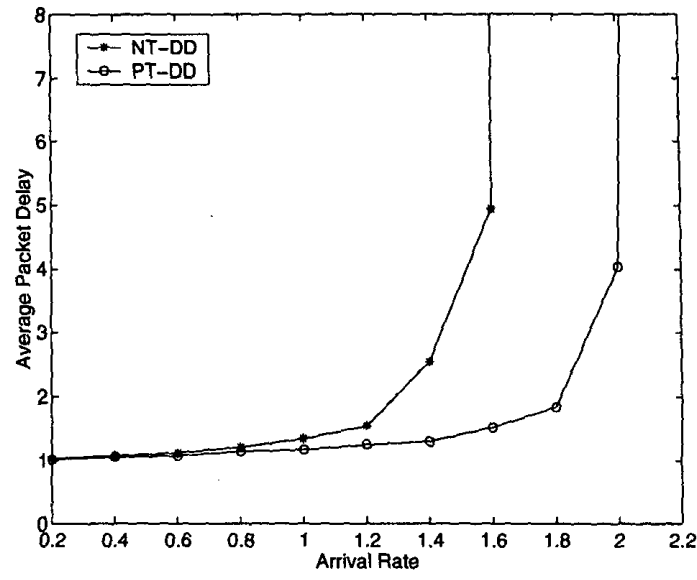

Figure 5: Pseudo-Bayesian stabilization of the RA scheme. Perfect tracking and decorrelator and matched filter for second stage.

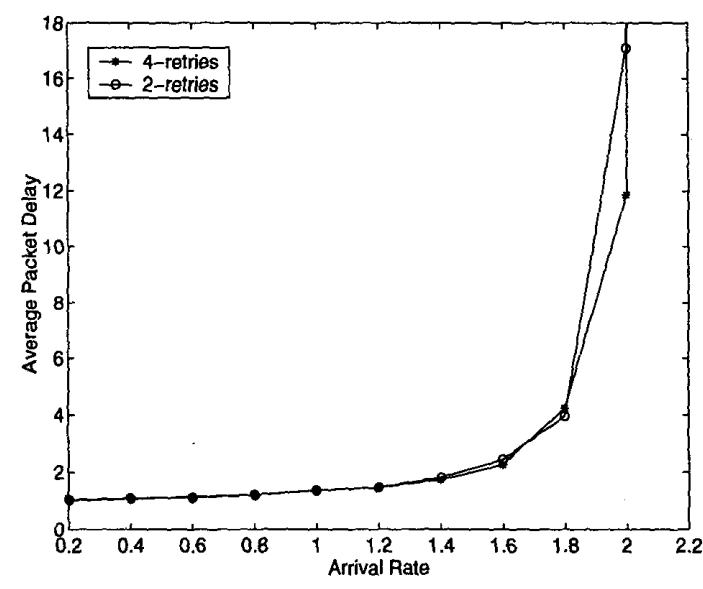

Figure 6: FCFS splitting for stabilization of the RA scheme. Perfect tracking and decorrelator for second stage. 